\title{
Asymmetry Assessment of Cardiac and Sympathetic Arms of the Baroreflex
}

\author{
Beatrice De Maria ${ }^{1}$, Vlasta Bari $^{2}$, Beatrice Cairo ${ }^{3}$, Emanuele Vaini $^{2}$, Elisabeth Lambert ${ }^{4}$, \\ Murray Esler ${ }^{5}$, Mathias Baumert ${ }^{6}$, Sergio Cerutti ${ }^{7}$, Laura Dalla Vecchia ${ }^{1}$, Alberto Porta ${ }^{2,3}$ \\ ${ }^{1}$ IRCCS Istituti Clinici Scientifici Maugeri, Italy \\ ${ }^{2}$ Department of Cardiothoracic, Vascular Anesthesia and Intensive Care, IRCCS Policlinico San \\ Donato, San Donato Milanese, Milan, Italy \\ ${ }^{3}$ Department of Biomedical Sciences for Health, University of Milan, Milan, Italy \\ ${ }^{4}$ Faculty of Health, Arts and Design, Swinburne University of Technology \\ ${ }^{5}$ Human Neurotransmitter Laboratory, Baker IDI Heart and Diabetes Institute, Melbourne, VIC, \\ Australia \\ ${ }^{6}$ School of Electrical and Electronic Engineering, University of Adelaide, Adelaide, SA, Australia \\ ${ }^{7}$ Department of Electronics Information and Bioengineering, Politecnico di Milano, Milan, Italy
}

\begin{abstract}
Pharmacological challenges allowed the characterization of the cardiac baroreflex $(c B R)$ asymmetry, defined as the difference of $c B R$ sensitivity $(c B R S)$ in response to arterial pressure $(A P)$ rises and fallings. Asymmetry of the sympathetic baroreflex (sBR) has never been explored. Two recently proposed analytical methods for $c B R S$ and $S B R$ sensitivity ( $S B R S$ ) estimation, i.e. the sequence (SEQ) and the bivariate phase rectified signal averaging (PRSA) methods, allow the noninvasive assessment of $c B R$ and $S B R$ asymmetry, respectively, from the spontaneous fluctuations of heart period in response to systolic AP changes and from spontaneous variability of sympathetic discharge in response to diastolic AP variations. In the present study we applied SEQ and PRSA methods with the aim at evaluating the $C B R$ and $S B R$ asymmetry in young healthy subjects during incremental head-up tilt. We found that sBRS computed by the SEQ method over negative diastolic AP changes was significantly more negative than that computed over positive AP diastolic changes. Asymmetric cBR behavior was not observed. In addition, we confirmed the superiority of the SEQ method compared to the PRSA one in assessing the asymmetry of baroreflex control. We conclude that in physiological conditions baroreflex asymmetry is a peculiar characteristic of $s B R$ and not of the $c B R$.
\end{abstract}

\section{Introduction}

The baroreflex (BR) is a complex physiological mechanism composed by different branches aiming at the maintenance of the arterial pressure (AP) towards nearly constant levels. The cardiac BR (cBR) branch reacts to $A P$ variations by varying the heart period (HP), while the sympathetic baroreflex (sBR) branch responds to AP changes by modulating the sympathetic activity. The characterization of $\mathrm{cBR}$ and $\mathrm{sBR}$ is usually based on the cBR and sBR sensitivity (cBRS and sBRS, respectively) estimation. The cBRS represents the variation of HP in response to a unit variation of systolic AP (SAP), while sBRS the variation of MSNA burst rate in response to a unit change of diastolic AP (DAP). cBRS and sBRS can be estimated noninvasively by exploiting the spontaneous variability of HP and SAP and of MSNA and DAP respectively [1-3]. Since both cBR and $\mathrm{sBR}$ react to either AP increases or decreases, some analytical methods have been recently proposed with the aim at separately computing the cBRS and sBRS driven by positive and negative AP variations $[3,4]$. These methods are suitable to assess the cBR and sBR asymmetry defined as the different magnitude of $\mathrm{cBR}$ and $\mathrm{sBR}$ response to positive and negative $\mathrm{AP}$ variations. The cBR asymmetry has been originally described in [5]: it was observed that cBRS in response to AP falling caused by the administration of amylnitrate was $40 \%$ of the cBRS induced by the AP rise induced by the administration of phenylephrine. Limited data are available about the BR asymmetry especially when evaluated over sBR and in physiological conditions.

The aim of this work is to characterize cBR and sBR asymmetry in young healthy subjects during incremental head-up tilt $[6,7]$. We exploited methods based on the spontaneous variability of HP, SAP, DAP and MSNA burst rate and able to separately compute cBRS and sBRS 
driven by positive and negative AP changes, namely the sequence (SEQ) [8] and bivariate phase rectified signal averaging (PRSA) methods $[9,10]$.

\section{Experimental protocol and data analysis}

\subsection{Experimental protocol}

The experimental protocol is fully described in [6]. The study protocol was approved by the Alfred Hospital Ethics Review Committee (n. 144/06) and conformed to the relevant guidelines of the National Health and Medical Research Council of Australia and to the principles of the Declaration of Helsinki for medical research involving humans. All subjects provided written informed consent. Briefly, 12 young healthy subjects (9 females; age from 20 to 36 years, median=22.5 years) were enrolled in the study. Each subject underwent incremental head-up tilt, starting from supine position. The subjects were sequentially tilted at $20^{\circ}, 30^{\circ}, 40^{\circ}$ and $60^{\circ}$. Sessions lasted 10 minutes each. We recorded lead III electrocardiogram (ECG), invasive AP from the radial artery and MSNA via a tungsten microelectrode inserted into the peroneal nerve. The raw MSNA signal was bandpass filtered $(700-2000 \mathrm{~Hz})$, amplified, rectified and integrated with a time constant of $0.1 \mathrm{~s}$, in order to obtain integrated MSNA signal. ECG, AP and integrated MSNA were sampled at $1000 \mathrm{~Hz}$.

\subsection{Beat-to-beat time series extraction}

For each cardiac beat, HP was calculated as the temporal distance between two consecutive R-wave peaks detected on the ECG. The maximum value of the AP within each $i$ th HP was taken as $i$ th SAP. The $i$ th DAP is the minimum of the AP before the $i$ th SAP. Calibrated MSNA series was extracted according to [7]. Briefly, the MSNA bursts were searched in a temporal window ranging from 0.9 to $1.7 \mathrm{~s}$ from the considered $\mathrm{R}$-wave peak, and detected using a running threshold following the baseline wandering and taking into account burst amplitude and noise level. Then, the detected bursts were counted in a moving time window of $5 \mathrm{~s}$ advancing at steps of $1 \mathrm{~ms}$. The counted MSNA signal was filtered with a cut-off frequency of $0.5 \mathrm{~Hz}$. Low-pass filtered counted MSNA signal was sampled at the occurrence of the first R-wave peak of the $i$ th HP and the values was divided by the frame length (i.e. $5 \mathrm{~s}$ ), thus obtaining the variability series of the number of MSNA burst per unit of time expressed in bursts $\cdot \mathrm{s}^{-1}$.

\section{3. $\quad$ cBR analysis methods}

The cBRS was estimated by the SEQ [8] and PRSA
$[9,10]$ methods. Briefly, the SEQ method is based on the search of 3 consecutive and contemporaneous increases or decreases of HP and SAP indicated as SEQ+/+ and SEQ-/- patterns. SEQ+/+ and SEQ-/- patterns were deemed to be of $\mathrm{cBR}$ origin regardless of the magnitude of the SAP and HP variations and the degree of HP-SAP correlation. The slope of the regression line calculated in the plane $[\operatorname{SAP}(i), \operatorname{HP}(i)]$, where $i$ the beat counter, was taken as cBRS estimate. The final cBRS estimate was calculated by separately averaging the slopes computed over all SEQ+/+ and SEQ-/- patterns [8]. cBRS was expressed in $\mathrm{ms}^{\cdot} \mathrm{mmHg}^{-1}$. The percentage of cBR sequences (SEQ\% $\%_{\mathrm{cBR}}$ ) was computed by separately considering SEQ+/+ and SEQ-/- patterns as well.

The PRSA method is based on the definition of the anchor point over the SAP series as the cardiac beat when $\triangle \operatorname{SAP}(i)=\operatorname{SAP}(i)-\operatorname{SAP}(i-1)>0 \quad[10]$. The pattern $\triangle \mathrm{SAP}(i)>0$ was labeled as PRSA + . For each anchor point, a segment over the HP series was selected, from $\operatorname{HP}(i-7)$ to $\mathrm{HP}(i+7)$. All the obtained segments were aligned and centered at the anchor points. cBRS was calculated as $[(X(0)+X(1)-X(-1)-X(-2)] / 4$, where $X(0), X(1), X(-1)$ and $\mathrm{X}(-2)$ were the means of HPs at $i, i+1, i-1$ and $i-2$ respectively. cBRS was also calculated by selecting as the anchor point the cardiac beat when $\triangle \operatorname{SAP}(i)<0$ [3]. The pattern $\triangle \mathrm{SAP}(i)<0$ was labeled as PRSA-. cBRS was expressed in ms. Normalized cBRS [9] (ncBRS) was obtained by dividing cBRS by the averaged $\triangle \mathrm{SAP}$ at the anchor point. ncBRS was expressed in $\mathrm{ms}^{\cdot} \mathrm{mmHg}^{-1}$. Since a negative cBRS computed over PRSA- indicated a working cBR, we reversed its sign.

\section{4. sBR analysis methods}

The sBRS was estimated by the SEQ [1] and PRSA [4] methods. Briefly, sBRS estimation by the SEQ method is based on the search of 3 consecutive and contemporaneous DAP increases and MSNA burst rate decreases or DAP decreases and MSNA burst rate increases indicated as SEQ+/- and SEQ-/+ patterns. The SEQ+/- and SEQ-/+ patterns were deemed to be of sBR origin regardless of the magnitude of the DAP and MSNA burst rate variations and the degree of MSNADAP correlation. The slope of the regression line calculated in the plane $[\mathrm{DAP}(i), \operatorname{MSNA}(i)]$ was taken as sBRS estimate. The final sBRS estimate was calculated by separately averaging the slopes computed over all SEQ+/- and SEQ-/+ patterns. sBRS was expressed in bursts $\cdot \mathrm{s}^{-1} \cdot \mathrm{mmHg}^{-1}$. The percentage of $\mathrm{sBR}$ sequences $\left(\mathrm{SEQ} \%_{\mathrm{sBR}}\right)$ was computed by separately considering SEQ+/- and SEQ-/+ patterns as well.

The PRSA method for sBRS estimation is a recently proposed adaptation of the original method for cBRS estimation [4]. The method was analogous to that utilized over HP and SAP but HP was substituted with MSNA variability and SAP with DAP variability. SBRS was 

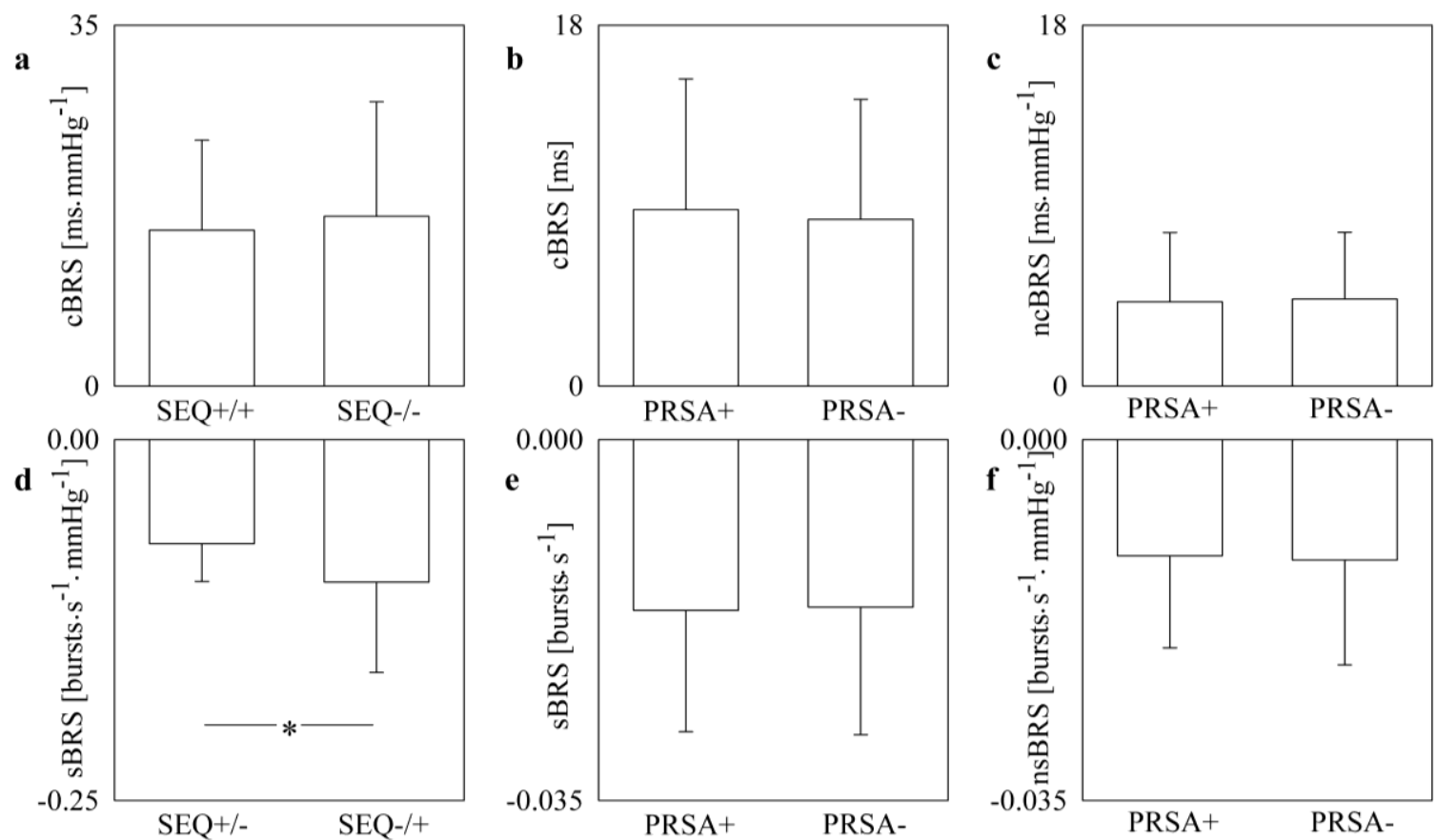

Figure 1. The error bar graphs show BRS estimates as computed via SEQ (a,b) and PRSA (c,d,e,f) methods as a function of the type of BR pattern, namely SEQ+/+ and SEQ-/- in (a) and SEQ+/- and SEQ-/+ in (b), and sign of the AP variation, namely PRSA+ and PRSA- in (c,d,e,f). BRS estimates calculated via PRSA method are reported in absolute (c,e) and normalized $(\mathrm{d}, \mathrm{f})$ values. Data are pooled together regardless of the experimental condition. The symbol $*$ indicates $p<0.05$.

expressed in bursts $\cdot \mathrm{s}^{-1}$, while normalized sBRS (nsBRS) in bursts $\cdot \mathrm{s}^{-1} \cdot \mathrm{mmHg}^{-1}$. Since a positive $\mathrm{sBRS}$ computed over PRSA+ indicated a working sBR, we reversed its sign.

\subsection{Statistical analysis}

After pooling together all the data regardless of the experimental condition, the differences between parameters computed within the same approach, but by separately considering positive and negative AP variation, were tested by means of paired t-test, or Wilcoxon signed rank test when appropriate. Data are reported as mean+standard deviation. A $p<0.05$ was always
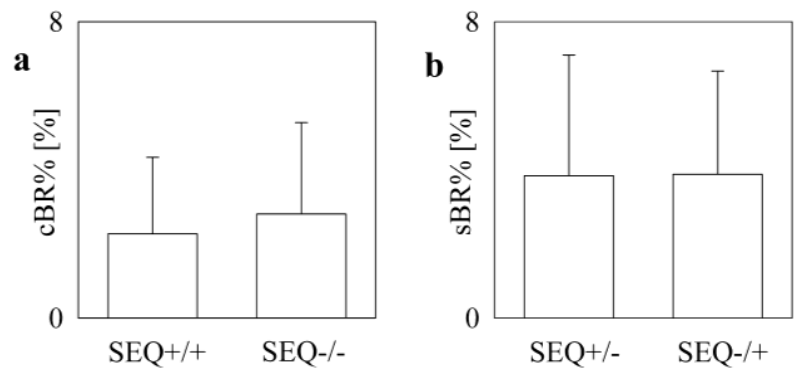

Figure 2. The error bar graphs show cBR\% (a) and sBR\% (b) as a function of the type of BR pattern detected by the SEQ method, namely SEQ+/+ and SEQ-/- in (a) and SEQ+/- and SEQ-/+ in (b). Data are pooled together regardless of the experimental condition. considered as significant.

\section{Results}

The bar graphs of Figure 1 show the cBRS (Figs.1a,b,c) and sBRS (Figs.1d,e,f) estimates derived by considering separately positive or negative AP variations. Data are pooled together regardless of the experimental condition. As to cBRS, no differences between cBRSs were observed and this result held regardless of the method. As to sBRS, the SEQ method found that sBRS computed over negative AP variations was significantly more negative than that calculated over positive AP changes (Fig.1d). Similar sBRSs were detected by the PRSA technique (Figs.1e,f).

Figure 2 shows cBR\% (Fig.2a) and sBR\% (Fig.2b) as a function of the type of the BR pattern detected by the SEQ method. The percentages of BR patterns featuring positive AP variations were similar to that exhibiting negative AP changes. This conclusion held for $c B R$ (Fig.2a) and sBR (Fig.2b). The percentages of AP variations over which the PRSA technique was grounded were close to $50 \%$ regardless of the sign of the AP variation and arm of the BR.

\section{Discussion}

We tested the hypothesis that BR exhibits a different 
behavior when solicited with positive and negative AP variations, referred to BR asymmetry. The analysis was carried out in physiological conditions and during the sympathetic activation evoked by a graded postural challenge (i.e. incremental head-up tilt). The BR asymmetry was studied over two different BR arms (i.e. cBR and sBR) and the BR sensitivity was considered as the target variable of the study.

We found that asymmetry is a peculiar aspect of the sBR, while cBR did not show an asymmetric behavior. The lack of cBR asymmetry is in contrast with findings present in literature reporting that $\mathrm{cBRS}$ is greater in response to AP rises $[5,11,12]$. Since this observation is based on a pharmacological stimulus able to evoke important AP changes [5,11,12], we conclude that the limited amplitude of the physiological SAP variations is not sufficient to explore this feature of the cBR. Conversely, the physiological variability of DAP is sufficient to detect the sBR asymmetry indicating that the same absolute variation of DAP produces more important sympathetic activation than inhibition via the sBR. In addition, we confirmed that the PRSA method is less powerful than the SEQ technique in describing the different $\mathrm{BR}$ responses to positive and negative $\mathrm{AP}$ changes [3]. Indeed, the sBR asymmetry was detected only by the SEQ method, while the PRSA technique hided this aspect. This result suggests that the SEQ method should be preferred when the BR asymmetry is the target. The good performance of SEQ method is likely to be related to its focus on particular rare patterns lasting several beats and probably of BR origin, while the PRSA uses basic AP variations from one beat to the next one that might not drive HP or MSNA responses.

\section{Conclusion}

In physiological conditions we described the asymmetric behavior of the BR in response to positive and negative AP changes. This behavior was evident over the sBR, while it was not visible over the cBR. We conclude that this peculiar nonlinear feature of the BR might produce nonlinear dynamics more evident over MSNA variability than HP series. Additional studies over pathological populations are needed to better understand the functional meaning of the observed behavior and whether the loss of this feature could represent a hallmark of pathology.

\section{References}

[1] A. Marchi, V. Bari, B. De Maria, M. Esler, E. Lambert, M. Baumert and A. Porta, "Simultaneous characterization of sympathetic and cardiac arms of the baroreflex through sequence techniques during incremental head-up tilt," Front. Physiol., vol. 7, art. no. 438, 29, 2016.
[2] G. D. Pinna, A. Porta, R. Maestri, B. De Maria, L. A. Dalla Vecchia and M. T. La Rovere, "Different estimation methods of spontaneous baroreflex sensitivity have different predictive value in heart failure patients," J. Hypertens., vol. 35, pp. 1666-1675, 2017.

[3] B. De Maria, V. Bari, M. Ranucci, V. Pistuddi, G. Ranuzzi, A. C. M. Takahashi, A. M. Catai, L. Dalla Vecchia, S. Cerutti and A. Porta, "Separating arterial pressure increases and decreases in assessing cardiac baroreflex sensitivity via sequence and bivariate phase-rectified signal averaging techniques," Med. Biol. Eng. Comput., in press, 2018.

[4] B. De Maria, V. Bari, B. Cairo, E. Vaini, E. Lambert, M. Esler, M. Baumert, S. Cerutti, L. Dalla Vecchia and A. Porta, "Estimating sympathetic baroreflex sensitivity via phase rectified signal averaging technique," Sixth National Italian Conference of Bioengineering, 2018.

[5] T. G. Pickering, B. Gribbin and P. Sleight, "Comparison of the reflex heart rate response to rising and falling arterial pressure in man," Cardiovasc. Res., vol. 6, pp. 277-283, 1972.

[6] E. Lambert, N. Eikelis, M. Esler, T. Dawood, M. Schlaich, R. Bayles, F. Socratous, A. Agrotis, G. Jennings, G. Lambert and G. Vaddadi, "Altered sympathetic nervous reactivity and norepinephrine transporter expression in patients with postural tachycardia syndrome," Circ. Arrhythm Electrophysiol., vol. 1, pp. 103-109, 2008.

[7] A. Marchi, V. Bari, B. De Maria, M. Esler, E. Lambert, M. Baumert and A. Porta, "Calibrated variability of muscle sympathetic nerve activity during graded head-up tilt in humans and its link with noradrenaline data and cardiovascular rhythms," Am. J. Physiol., vol. 310, pp. R1134-R1143, 2016.

[8] G. Bertinieri, M. di Rienzo, A. Cavallazzi, A. U. Ferrari, A. Pedotti and G. Mancia, "A new approach to analysis of the arterial baroreflex," J. Hypertens. Suppl., vol. 3, pp. S79S81, 1985.

[9] A. Muller, A. Morley-Davies, P. Barthel, K. Hnatkova, A. Bauer, K. Ulm, M. Malik and G. Schmidt, "Bivariate phase-rectified signal averaging for assessment of spontaneous baroreflex sensitivity: normalization of the results," J. Electrocardiol., vol. 45, pp. 77-81, 2012.

[10] A. Bauer, A. Morley-Davies, P. Barthel, A. Muller, K. Ulm, M. Malik and G. Schmidt, "Bivariate phase-rectified signal averaging for assessment of spontaneous baroreflex sensitivity: pilot study of the technology," J. Electrocardiol., vol. 43, pp. 649-653, 2010.

[11] J. Parlow, J. P. Viale, G. Annat, R. Hughson and L. Quintin, "Spontaneous cardiac baroreflex in humans. Comparison with drug-induced responses," Hypertension, vol. 25, pp. 1058-1068, 1995.

[12] G. Parati, M. Di Rienzo, G. Bertinieri, G. Pomidossi, R. Casadei, A. Groppelli, A. Pedotti, A. Zanchetti and G. Mancia, "Evaluation of the baroreceptor-heart rate reflex by 24-hour intra-arterial blood pressure monitoring in humans," Hypertension, vol. 12, pp. 214-222, 1988.

Address for correspondence.

Beatrice De Maria,

IRCCS Istituti Clinici Scientifici Maugeri, Via Camaldoli 64, 20138 Milano, Italy

Tel: +390250725286

Email: beatrice.demaria@icsmaugeri.it 